\title{
Unscented Kalman Filtering of a Simulated pH System
}

\author{
Andrey Romanenko,* Lino O. Santos, and Paulo A. F. N. A. Afonso \\ GEPSI-PSE Group, Departamento de Engenharia Química, Universidade de Coimbra, Pinhal de Marrocos, \\ Pólo II, 3030 Coimbra, Portugal
}

\begin{abstract}
Recently, the unscented Kalman filter (UKF) algorithm, which is a new generalization of the Kalman filter for nonlinear systems, was proposed in the literature. It has significant advantages over its widely used predecessor, the extended Kalman filter (EKF). These include better accuracy and simpler implementation and the dispensability of system and measurement model differentiability. In this work, we compare the performance of the two approaches in a simulated $\mathrm{pH}$ process with three situations considered. The first one evaluates the performance differences between the unscented transform and the EKF linearization, as applied to the nonlinear $\mathrm{pH}$ output equation. In the second simulation, the complete filter algorithms are compared in a state estimation framework. The third case concerns parameter estimation. In all three cases, the UKF produced more-accurate results.
\end{abstract}

\section{Introduction}

State and parameter estimation has been used widely for the purpose of control and monitoring. ${ }^{1}$ One of the most-utilized linear estimation methods is the Kalman filter, ${ }^{2}$ whereas nonlinear problems may be tackled with the extended Kalman filter $(\mathrm{EKF}){ }^{3}$

The EKF contains several flaws that may seriously affect its performance. For instance, although the a priori state mean may be estimated propagating the nonlinear system directly, the corresponding covariance estimate calls for the calculation of the state transition matrix, which, in turn, requires linearization of the system model. This may introduce significant errors if the error in the state estimate is not sufficiently small. Indeed,

$$
\boldsymbol{P}_{k \mid k+1}=\Phi \boldsymbol{P}_{k \mid k} \Phi^{\mathrm{T}}+\boldsymbol{D} \boldsymbol{Q}_{k} \boldsymbol{D}^{\mathrm{T}}
$$

where $\Phi$ is the state transition matrix. For timeinvariant systems, it is

$$
\Phi=\mathrm{e}^{F \Delta t}
$$

where $F$ is the Jacobian of the continuous system and $\Delta t$ is the time interval. There are continuous time versions of the Kalman filter where the state and its covariance matrix are integrated directly. However, this results in a large system of ordinary differential equations (ODEs) to be integrated. Besides, discrete time formulation is a natural form for implementation in modern digital control and measurement systems. Moreover, the matrix exponential evaluation is a potentially ill-conditioned operation. ${ }^{4}$ Furthermore, differentiability of the system and measurement models is required. Generally, various cases of nondifferentiable systems have required special treatment from a control standpoint, such as friction compensation in control valves, ${ }^{5}$ piece-wise switching of parameters in process models, ${ }^{6}$ and physical configuration. ${ }^{7}$ Many control and optimization algorithms make an assumption of smoothness or

* To whom correspondence should be addressed. Tel: +351239-798700. Fax: +351-239-798703.E-mail: andrew@eq.uc.pt. "well behavior" of functions they involve and approximate them as such.

An enhanced filtering technique that effectively addresses the aforementioned issues was proposed in the literature; ${ }^{8}$ this technique is called the unscented Kalman filter (UKF) method. Theoretical treatment of the UKF formalism may be found elsewhere. ${ }^{9-12}$ Many applications utilizing the UKF have been reported since that time. Note that most of them are in the area of aerospace navigation and tracking, where one frequently encounters severe nonlinearity and fast dynamics and where the UKF was developed. For example, faster convergence is achieved by the UKF in a spacecraft attitude estimation framework. ${ }^{13} \mathrm{~A}$ comparison of nonlinear filters for tracking a ballistic object ${ }^{14}$ also favors this new technique. The ability of the UKF to address discontinuous systems resulted in a successful application of the UKF to models with freeplay and hysteresis..$^{15}$ Superior performance of the UKF, as compared to the EKF, has been observed in communication systems. ${ }^{16}$ This technique has also been integrated with an interactive multiple model algorithm in a maneuvering target tracking framework. ${ }^{17}$ Other application areas include on-line training of neural networks. ${ }^{18,19}$ The latter work also addresses the use of the UKF for system identification and simultaneous state and parameter estimation.

Despite the apparent advantages of the UKF over the $\mathrm{EKF}$, the former has not been used widely in chemical process engineering and most relevant applications reported in the literature are the state estimation of a biological process and partitioned filtering, as a means of system identification from simulated chaotic data. ${ }^{21}$ As with the EKF in the $1980 \mathrm{~s}, 22$ this may be due to the unfamiliarity of researchers and practitioners with this tool.

In a previous work, the performance of the UKF and the EKF were compared in the state estimation of a simulated continuously stirred tank reactor (CSTR) system exhibiting strong nonlinearity. ${ }^{23}$ Although the focus of that study was the nonlinearity of the system equations, the measurements were linear, with respect to the states. However, some chemical engineering systems feature highly nonlinear measurements, and, 
thus, they may also benefit from the use of the UKF. In the present work, we apply both the UKF and the $\mathrm{EKF}$ to a simulated $\mathrm{pH}$ system to assess their performance. While its state equations are still nonlinear, this nonlinearity is rather weak, compared to that of the output equation. Besides, its output Jacobian is an expression of significant size and complexity.

The paper is organized as follows: the UKF algorithm is described in Section 2, and the system under study and the simulation setup are outlined in Section 3. Section 4 presents obtained results and a comparison of the filter performance by simulation. Finally, conclusions are drawn in Section 5.

\section{The UKF Algorithm}

Consider a dynamic system approximated in discrete time as

$$
\boldsymbol{x}_{k}=f\left(\boldsymbol{x}_{k-1}, \boldsymbol{u}_{k-1}, \boldsymbol{v}_{k-1}, k-1\right)
$$

with a measurement vector

$$
\boldsymbol{y}_{k}=h\left(\boldsymbol{x}_{k}, \boldsymbol{u}_{k}, \omega_{k}, k\right)
$$

In eqs 1 and $2, \boldsymbol{x}$ is the state vector, $\boldsymbol{u}$ the control input vector, $v$ the system noise vector, $\omega$ the measurement noise vector, $f(\cdots)$ the process model, and $h(\cdots)$ the measurement model.

A Kalman-type filter consists of two major steps: prediction, in which a new a priori estimate of the state vector is computed using the system model; and correction, in which this predicted estimated is improved using available measurements. This structure also is maintained in the UKF. However, instead of system and measurement model truncation, the probability densities are truncated. ${ }^{24}$

The unscented transformation ${ }^{8,25}$ makes use of deterministically chosen sigma points that are propagated through the original nonlinear system equations. Using an appropriate weighing scheme, it is then possible to calculate the statistical properties of the propagated point set with the same performance as the truncated second-order Gaussian filter but without the need to calculate the system's Jacobians or Hessians. ${ }^{9}$ Related approaches may be found elsewhere. . $^{7,10}$

In the UKF formulation, the system state vector is augmented,

$$
\boldsymbol{x}_{k-1 \mid k-1}^{\mathrm{a}} \triangleq\left(\left.\boldsymbol{x}_{k-1}\right|_{k-1} \quad \boldsymbol{v}_{k-1} \quad \boldsymbol{\omega}_{k-1}\right)
$$

and the covariance of the augmented state vector is given by

$$
\boldsymbol{P}_{k-1 \mid k-1}^{\mathrm{a}}=\left[\begin{array}{lll}
\left.\boldsymbol{P}_{k-1}\right|_{k-1} & 0^{n \times q} & 0^{n \times r} \\
0^{q \times n} & \boldsymbol{Q}_{k-1} & \boldsymbol{P}_{k-1}^{v \omega} \\
0^{r \times n} & \boldsymbol{P}_{k-1}^{\omega \nu} & \boldsymbol{R}_{k-1}
\end{array}\right]
$$

where $n$ is the dimension of the original state vector, and $q$ and $r$ are the sizes of the covariance matrixes of the system and measurement noise, respectively. $\boldsymbol{P}_{k-1}^{v \omega}$ and $\boldsymbol{P}_{k-1}^{\omega \nu}$ are the correlation matrixes between the system and measurement noises. The dimension of the resulting augmented state vector is

$$
n^{\mathrm{a}}=n+q+r
$$

Consequently, a set of $2 n^{\mathrm{a}}+1$ symmetric sigma points is computed:

$$
\begin{aligned}
& X_{k-1 \mid k-1}^{*}= \\
& \quad\left[0, \sqrt{\left(n^{\mathrm{a}}+\kappa\right) \boldsymbol{P}_{k-1 \mid k-1}^{a}},-\sqrt{\left(n^{\mathrm{a}}+\kappa\right) \boldsymbol{P}_{k-1 \mid k-1}^{\mathrm{a}}}\right]
\end{aligned}
$$

where $\kappa$ is a parameter for "fine-tuning" the higher-order moments of the approximation. For any symmetric prior distribution with kurtosis $k$, if $\kappa$ is chosen such that 0 $<n^{\mathrm{a}}+\kappa \leq k$, then the predictions of means and covariances are more accurate than those of the EKF. Note that the unscented transformation properties hold for any choice of the matrix square root in eq 6 . However, for numerical robustness and computational efficiency reasons, the Cholesky decomposition should be preferred. ${ }^{8}$

Because the sigma point set $X^{*}$ is zero mean, the estimate $\hat{\boldsymbol{x}}_{k-1 \mid k-1}^{\mathrm{a}}$ is added to each sigma point in vector $x_{k-1 \mid k-1}^{*}$ and the resulting vector of sigma points is

$$
\begin{aligned}
& X_{k-1 \mid k-1}= \\
& \quad\left[X_{k-1 \mid k-1,1}^{*}+\hat{\boldsymbol{x}}_{k-1 \mid k-1}^{\mathrm{a}}, \ldots, X_{k-1 \mid k-1,2 n+1}^{\mathrm{a} *}+\hat{\boldsymbol{x}}_{k-1 \mid k-1}^{\mathrm{a}}\right]
\end{aligned}
$$

The prediction step consists of (i) the mapping of the sigma point set through the nonlinear system

$$
\mathscr{X}_{k \mid k-1}=f\left(\mathscr{X}_{k-1 \mid k-1}, u_{k-1}, k-1\right)
$$

and (ii) the calculation of the predicted mean as

$$
\hat{\boldsymbol{x}}_{k \mid k-1}=\sum_{i=1}^{2 n^{\mathrm{a}+1}} W_{i} \mathscr{X}_{i, k \mid k-1}
$$

where $W$ is a weight vector of size $2 n^{a}+1$ with elements

$$
W_{i}= \begin{cases}\frac{\kappa}{n^{\mathrm{a}}+\kappa} & (\text { if } i=1) \\ \frac{1}{2\left(n^{\mathrm{a}}+\kappa\right)} & (\text { otherwise }(\text { if } i \neq 1))\end{cases}
$$

The predicted covariance is computed as

$$
\begin{aligned}
\boldsymbol{P}_{k \mid k-1}=\sum_{i=1}^{2 n^{\mathrm{a}}+1} W_{i}\left[\mathscr{X}_{i, k \mid k-1}-\hat{\boldsymbol{x}}_{k \mid k-1}\right] \times \\
{\left[\mathscr{X}_{i, k \mid k-1}-\hat{\boldsymbol{x}}_{k \mid k-1}\right]^{\mathrm{T}} }
\end{aligned}
$$

The statistics of the innovation is determined in a similar manner. The predicted observation is computed as

$$
\begin{gathered}
Y_{k}=h\left(X_{i, k \mid k-1}, u_{k}, k\right) \\
\hat{\boldsymbol{y}}_{k}=\sum_{i=1}^{2 n^{\mathrm{a}+1}} W_{i} \mathscr{Y}_{i, k}^{a}
\end{gathered}
$$

and its covariance is determined as

$$
\boldsymbol{P}_{y}=\sum_{i=1}^{2 n^{\mathrm{a}+1}} W_{i} \mathscr{Y}_{i, k}-\hat{\boldsymbol{y}}_{k}\left[\mathscr{Y}_{i, k}-\hat{\boldsymbol{y}}_{k}\right]^{\mathrm{T}}
$$

The cross-correlation between the state estimate and the measurement sequence is 


$$
\boldsymbol{P}_{x y}=\sum_{i=1}^{2 n^{\mathrm{a}}+1} W_{i}\left[\mathscr{X}_{i, k \mid k-1}-\hat{\boldsymbol{x}}_{k \mid k-1}\right]\left[\mathscr{Y}_{i, k}-\hat{\boldsymbol{y}}_{k}\right]^{\mathrm{T}}
$$

The following equations are typical Kalman filter expressions for the measurement update. The Kalman gain is

$$
K_{k}=\boldsymbol{P}_{x y} \boldsymbol{P}_{y}^{-1}
$$

the updated mean of the state vector is

$$
\hat{\boldsymbol{x}}_{k \mid k}=\hat{\boldsymbol{x}}_{k \mid k-1}+\boldsymbol{K}_{k}\left(\boldsymbol{y}_{k}-\hat{\boldsymbol{y}}_{k}\right)
$$

and the corresponding covariance matrix is given by

$$
\boldsymbol{P}_{k \mid k}=\boldsymbol{P}_{k \mid k-1}-\boldsymbol{K}_{k} \boldsymbol{P}_{y} \boldsymbol{K}_{k}^{\mathrm{T}}
$$

The aforementioned algorithm describes the "standard" unscented Kalman filter. However, some enhancements have been reported recently in the literature, such as the use of additional points ${ }^{26}$ and a higher-order formulation ${ }^{27}$ for improving the accuracy of the filter, the reduction of the sigma point number in real-time applications, ${ }^{28}$ the scaling of the sigma point set to decrease the effect of sampling of nonlocal effects in high dimension state space, ${ }^{29}$ and a square-root formulation improving the numerical stability of the algorithm and decreasing its computational burden. ${ }^{30}$ Nevertheless, these advances address either implementation issues or particular systems and, therefore, are out of scope of this work. Instead, our goal is to illustrate that, even with the standard formulation of the UKF, it is possible to achieve performance improvements in a chemical process with output nonlinearity.

\section{System Description}

To compare the performance of each of the filters, we apply them to a simulated system reported by Biagiola and Figueroa that consists of a continuous stirred tank reactor (CSTR) in which a neutralization reaction between a strong acid (HA) and a strong base $(\mathrm{BOH})$ occurs in the presence of a buffer agent (BX) (see Figure 1). The motivation behind the choice of the present benchmark system is, on one hand, the simplicity of the system equations and, on the other hand, the complexity and nonlinearity of the output equation, especially of its Jacobian.

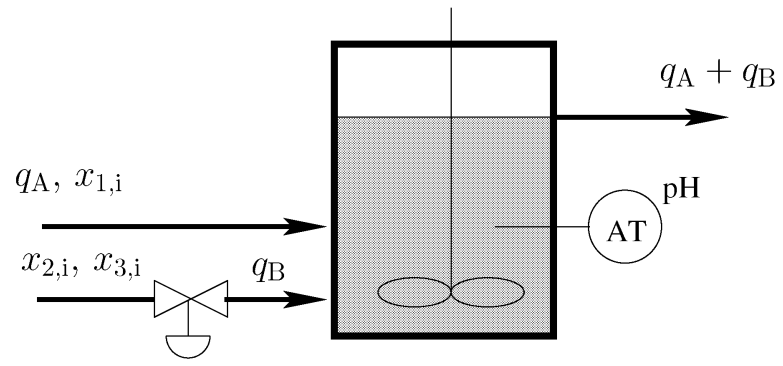

Figure 1. Neutralization process.
The state vector is defined as

$$
\begin{aligned}
& x_{1}=\left[\mathrm{A}^{-}\right] \\
& x_{2}=\left[\mathrm{B}^{+}\right]
\end{aligned}
$$

and

$$
x_{3}=\left[\mathrm{X}^{-}\right]
$$

where $\left[\mathrm{A}^{-}\right],\left[\mathrm{B}^{+}\right]$, and $\left[\mathrm{X}^{-}\right]$are the concentrations of the acid, base, and the buffer agent, respectively. The process model has the following form:

$$
\begin{gathered}
\dot{x}_{1}=\frac{1}{\theta}\left(x_{1, \mathrm{i}}-x_{1}\right)-\frac{1}{V} x_{1} q_{\mathrm{B}} \\
\dot{x}_{2}=-\frac{1}{\theta} x_{2}+\frac{1}{V}\left(x_{2, \mathrm{i}}-x_{2}\right) q_{\mathrm{B}} \\
\dot{x}_{3}=-\frac{1}{\theta} x_{3}+\frac{1}{V}\left(x_{3, \mathrm{i}}-x_{3}\right) q_{\mathrm{B}}
\end{gathered}
$$

$F(x, \xi) \equiv$

$$
\xi+x_{2}+x_{3}-x_{1}-\frac{K_{\mathrm{w}}}{\xi}-\frac{x_{3}}{1+\left(K_{\mathrm{x}} \xi / K_{\mathrm{w}}\right)}=0
$$

where $\xi=10^{-\mathrm{pH}}$ and $\theta=V / q_{\mathrm{A}}$. The terms $q_{\mathrm{A}}$ and $q_{\mathrm{B}}$ represent the inlet flow rates, $x_{1, \mathrm{i}}, x_{2, \mathrm{i}}$, and $x_{3, \mathrm{i}}$ are the inlet concentrations of the species, and $K_{\mathrm{x}}$ and $K_{\mathrm{w}}$ are the dissociation constants of the buffer and water, respectively. The system parameters used in this work are summarized in Table 1.

Expression 23 was rewritten as a third-order polynomial, ${ }^{31}$

$$
\begin{aligned}
\xi^{3}+\left[\frac{K_{\mathrm{w}}}{K_{\mathrm{x}}}+x_{3}+x_{2}-x_{1}\right] & \xi^{2}+ \\
& \left(x_{2}-x_{1}-K_{\mathrm{x}}\right) \frac{K_{\mathrm{w}}}{K_{\mathrm{x}}} \xi-\frac{K_{\mathrm{w}}^{2}}{K_{\mathrm{x}}}=0
\end{aligned}
$$

from which the value of the output may be derived by introducing the following auxiliary variables:

$$
\begin{gathered}
a=1 \\
b=\frac{K_{\mathrm{w}}}{K_{\mathrm{x}}}+x_{3}+x_{2}-x_{1} \\
c=\left(x_{2}-x_{1}-K_{\mathrm{x}}\right) \frac{K_{\mathrm{w}}}{K_{\mathrm{x}}} \\
d=-\frac{K_{\mathrm{w}}^{2}}{K_{\mathrm{x}}} \\
p=-\frac{b}{3 a} \\
q=p^{3}+\frac{b c-3 a d}{6 a^{2}} \\
r=\frac{c}{3 a}
\end{gathered}
$$

Solving for $\mathrm{pH}$, 


$$
\begin{aligned}
\mathrm{pH}=-\log \left\{\left[q+\sqrt{q^{2}+\left(r-p^{2}\right)^{3}}\right]^{1 / 3}+\right. \\
{\left.\left[q-\sqrt{q^{2}+\left(r-p^{2}\right)^{3}}\right]^{1 / 3}+p\right\} }
\end{aligned}
$$

The output equation (eq 25) clearly exhibits a very strong nonlinear behavior. To illustrate this phenomenon, the titration curve of the process is given in Figure 2 for $q_{\mathrm{B}}$ from $0 \mathrm{~L} / \mathrm{min}$ to $5 \mathrm{~L} / \mathrm{min}$, which is the region of very pronounced nonlinearity. The reader is referred elsewhere ${ }^{31}$ for the titration curve for a wider range of the inlet flow rates.

\section{Simulation Results}

To compare the filters, three simulation situations are considered. The first simulation evaluates the performance differences of the unscented transform and the EKF linearization as applied to the output (eq 25). In the second simulation, the complete filter algorithms are compared in a state estimation framework of the above system. A parameter estimation application is addressed in the third case.

4.1. Unscented Transform versus Linearization. This simulation consists of propagating a Gaussian random variable through a nonlinear function using the unscented transform and the linearization utilized in the Kalman filter. Namely, we map the state vector $\boldsymbol{x}$ through eq 25. To focus on a nonlinear region, the sample mean is chosen to be

$$
\overline{\boldsymbol{x}}=\left[\begin{array}{l}
9.3 \times 10^{-4} \\
5.4 \times 10^{-4} \\
4.3 \times 10^{-4}
\end{array}\right]
$$

which results in $\mathrm{pH}=6.13$, where the nonlinear behavior of the titration curve is evident (see Figure 2). The sample covariance is a diagonal matrix

$$
\boldsymbol{P}_{\mathrm{x}}=\operatorname{diag}\left\{\begin{array}{lll}
\sigma^{2} & \sigma^{2} & \sigma^{2}
\end{array}\right\}
$$

with $\sigma^{2}$ ranging from $2 \times 10^{-11}$ to $2 \times 10^{-9}$.

The linearization algorithm consists of calculating the propagated mean as

$$
\overline{\boldsymbol{y}}=h(\overline{\boldsymbol{x}})
$$

and the propagated covariance as

$$
\boldsymbol{P}_{y}=H P_{x} H^{T}
$$

where

$$
H=\left.\frac{\partial h}{\partial \mathbf{x}}\right|_{x=\bar{x}}
$$

Such an approach works well for linear systems. However, in nonlinear cases, the propagation of a Gaussian random variable produces a non-Gaussian variable. Note that the Jacobian $H$ of eq 25 is an expression of

Table 1: Model Parameters

\begin{tabular}{cll}
\hline symbol & \multicolumn{1}{c}{ parameter } & \multicolumn{1}{c}{ value } \\
\hline$x_{1, \mathrm{i}}$ & acid inlet concentration & $1.2 \times 10^{-3} \mathrm{~mol} / \mathrm{L}$ \\
$x_{2, \mathrm{i}}$ & base inlet concentration & $2.0 \times 10^{-3} \mathrm{~mol} / \mathrm{L}$ \\
$x_{3, \mathrm{i}}$ & buffer inlet concentration & $2.5 \times 10^{-3} \mathrm{~mol} / \mathrm{L}$ \\
$K_{x}$ & buffer dissociation constant & $10^{-7} \mathrm{~mol} / \mathrm{L}$ \\
$K_{\mathrm{w}}$ & water dissociation constant & $10^{-14} \mathrm{~mol}^{2} / \mathrm{L}^{2}$ \\
$V$ & reactor volume & $2.5 \mathrm{~L}$
\end{tabular}

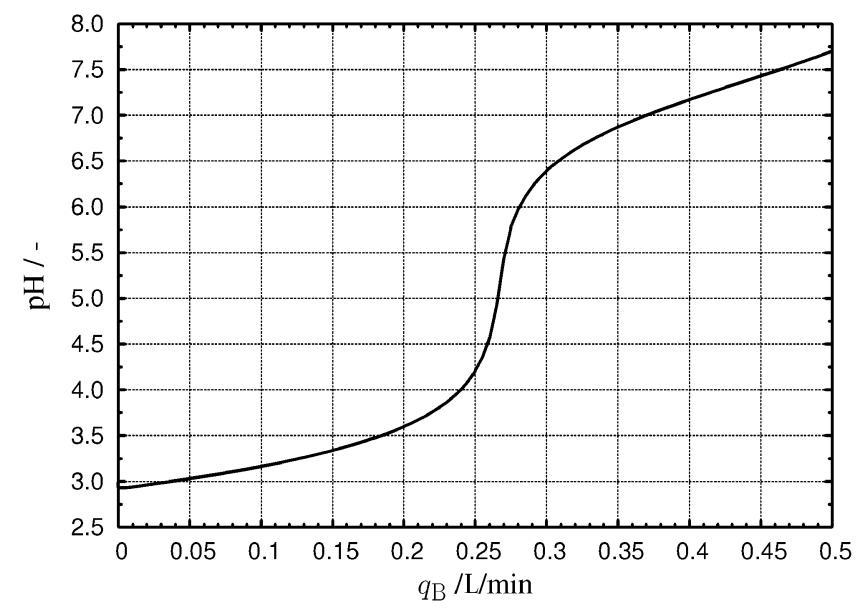

Figure 2. Titration curve.

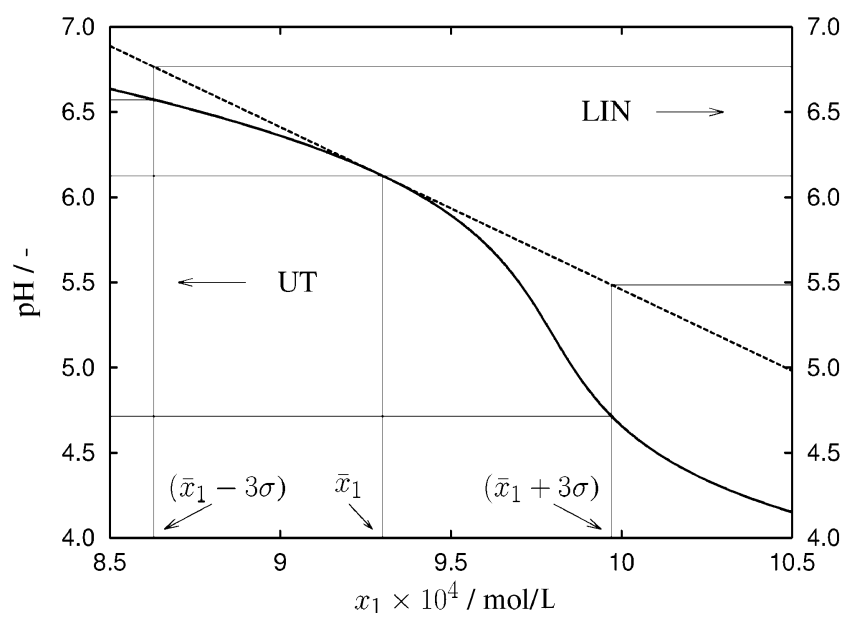

Figure 3. Transformation approaches.

significant complexity and size. Therefore, it is omitted from the article, for the sake of space.

On the contrary, the unscented transform does not make use of the Jacobian. Now that the dimension of the state vector is 3 , the sigma set is comprised of seven elements. In addition, for the reason of Gaussianity of $\boldsymbol{x}$, the parameter $\kappa$ is set to 0 , and the statistical properties of $\boldsymbol{y}$ are calculated with eqs $12-14$ and eq 10.

To enable a graphical interpretation of the differences between the two approaches, we analyze a simplified version of eq 25 in which $x_{2}$ and $x_{3}$ are kept constant at $5.4 \times 10^{-4} \mathrm{~mol} / \mathrm{L}$ and $4.3 \times 10^{-4} \mathrm{~mol} / \mathrm{L}$, respectively. In addition, $x_{1}$ is assumed to be a random variable with a mean of $\bar{x}_{1}=9.3 \times 10^{-4} \mathrm{~mol} / \mathrm{L}$ and variance of $\sigma^{2}=5 \times$ $10^{-10} \mathrm{~mol}^{2} / \mathrm{L}^{2}$. Such simplification is motivated solely by the fact that univariate functions are easier to analyze graphically. The solid line in Figure 3 corresponds to the resulting function for $x_{1} \in\left[8.5 \times 10^{-4}, 10.5\right.$ $\left.\times 10^{-4}\right] \mathrm{mol} / \mathrm{L}$, whereas the dashed straight line corresponds to the linearization of the function at $x_{1}=\bar{x}_{1}=$ $9.3 \times 10^{-4}$. The slope of this line is equal to the value of the first element of Jacobian $H$. Consequently, we form a set of points $\left[\left(\bar{x}_{1}-3 \sigma\right), \bar{x}_{1},\left(\bar{x}_{1}+3 \sigma\right)\right]$ and map them using eq 25.

In the unscented transform, these points are mapped using the actual nonlinear function (see the left vertical axis of Figure 3), and the resulting points are weighed to calculate the statistical properties of the result. In the (extended) linearization approach, only the prior 
Table 2: Univariate Transformation Results

\begin{tabular}{lccc}
\hline parameter & $\begin{array}{c}\text { Monte Carlo, } \\
\text { MC }\end{array}$ & $\begin{array}{c}\text { linearization, } \\
\text { LIN }\end{array}$ & $\begin{array}{c}\text { unscented transform, } \\
\text { UT }\end{array}$ \\
\hline mean & 6.0765 & 6.1252 & 6.0748 \\
variance & 0.070755 & 0.045471 & 0.070087
\end{tabular}

mean is propagated through the original function but the covariance is propagated through a linearized system (Jacobian). In this example, it is equivalent to the mapping of the other two points using the straight line (see the right vertical axis of Figure 3 ). This proves the fact that, in the (extended) linearization, the mean of the result is independent of the prior variance. Moreover, the variance of the result is not sensitive to the nonlinearity of the function in question. In this example, such linearization assumptions worsen the results. On the other hand, the unscented transform is able to predict the statistical properties quite well (see Table 2).

As in the univariate case previously mentioned, a similar performance difference is observed when all elements of $\boldsymbol{x}$ are considered random. The top plot of Figure 4 illustrates the residuals

$$
\tilde{y}=\bar{y}-\bar{y}_{\mathrm{T}}
$$

where $\bar{y}$ is the true mean and $\bar{y}_{\mathrm{T}}$ is the mean calculated by the transformations. The evolution of the true and predicted variance of $y$ is depicted in the bottom plot of Figure 4. The true statistical properties of the propagated variable were determined in a series of Monte Carlo runs, each consisting of $10^{6}$ samples.

Note that an increase in the input covariance $(\sigma)$ leads to higher prediction errors of both filters. However, one may observe two distinct regions of the residual plot: for $\sigma<4 \times 10^{-10} \mathrm{~mol}^{2} / \mathrm{L}^{2}$, the unscented transformation is substantially more accurate than its linearization counterpart, whereas, at higher values of the input covariance, the errors of both transformations are comparable. However, the variance predicted by the linearization approach is clearly underestimated.

The aforementioned test is repeated with the sample mean,

$$
\overline{\boldsymbol{x}}=\left[\begin{array}{l}
9.484 \times 10^{-4} \\
4.194 \times 10^{-4} \\
5.242 \times 10^{-4}
\end{array}\right]
$$

corresponding to $\mathrm{pH}=5$, which is the point of the highest sensitivity and nonlinearity of the output equation. The sample covariance is given by eq 27 . Figure 5 illustrates the residual and variance of the transformed random variable as a function of the sample covariance. It is evident that the mean prediction error of both approximations is lower than in the previous case, although the unscented transform remains more accurate. This may be explained by the fact that, in this region, the nonlinearity is almost symmetrical and, therefore, the linearization is able to achieve a satisfactory level of accuracy. This notwithstanding, the variance of the mapped variable is significantly overestimated, because the linearization does not take into account the nonlinear change in the derivative of the function in the vicinity of the nominal operating point. Such behavior in the calculation of the predicted output covariance of a Kalman-type filter may lead to a lower value of the Kalman gain and, thereby, the effect of the
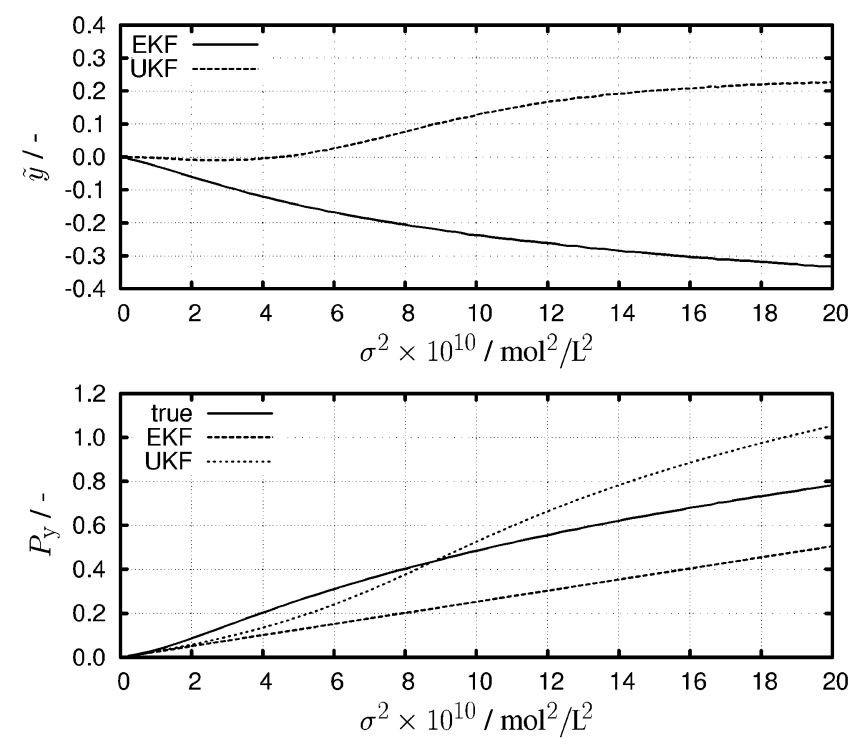

Figure 4. Transformation results in the vicinity of $\mathrm{pH}=6.13$ : mean (top) and covariance (bottom).
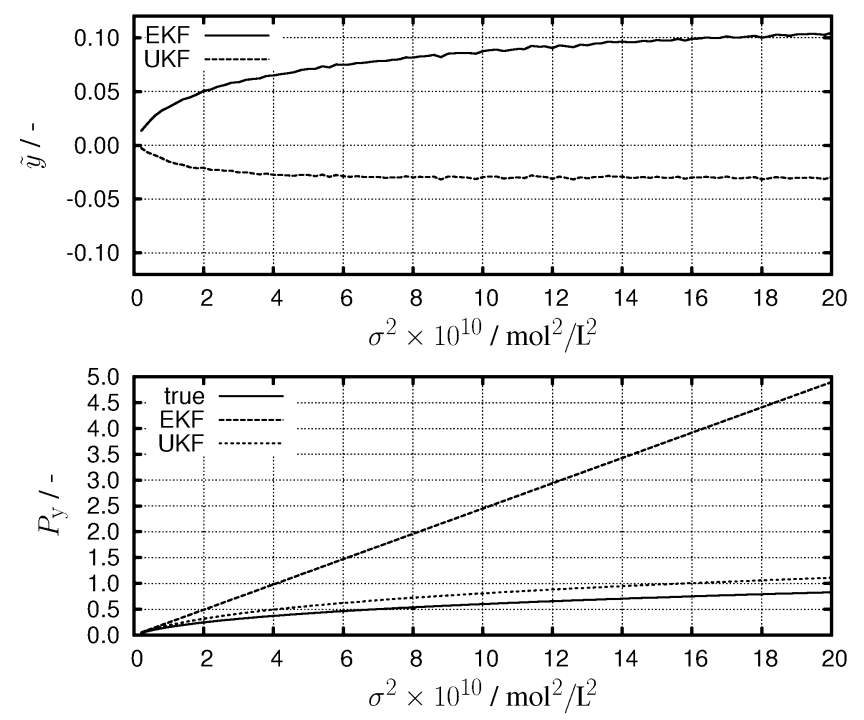

Figure 5. Transformation results in the vicinity of $\mathrm{pH}=5$ : mean (top) and covariance (bottom).

measurement update step of the observer may be undermined.

It is remarkable that the unscented transformation manifests smaller estimation errors over the entire range of the input covariance in both sets of nominal operating conditions.

4.2. State Estimation. Previously, it was shown how estimation results of a nonlinear map might be improved with the use of the unscented transform. In this subsection, we compare the accuracy of EKF and UKF, based on linearization and the unscented transformation, respectively. The system defined by eqs $20-22$ is approximated in discrete time as

$$
\boldsymbol{x}_{k}=f\left(\boldsymbol{x}_{k-1}, \boldsymbol{u}_{k-1}\right)+\boldsymbol{v}_{k-1}
$$

where $\boldsymbol{v}$ is the zero-mean Gaussian system noise with covariance 


$$
\boldsymbol{Q}=\operatorname{diag}\left\{\begin{array}{l}
2 \times 10^{-11} \\
2 \times 10^{-11} \\
2 \times 10^{-11}
\end{array}\right\} \mathrm{mol}^{2} / \mathrm{L}^{2}
$$

In addition, the measurement model has the form

$$
\boldsymbol{y}_{k}=h\left(\boldsymbol{x}_{k}\right)+\omega_{k}
$$

where $\omega_{k}$ is the zero-mean Gaussian measurement noise with a variance of $R=10^{-4}$. In addition, the system and measurement noise processes are not correlated. Although such system is, in fact, simpler than eqs 1 and 2 , these assumptions are fairly common in practice. ${ }^{32}$

The only measurement available is $\mathrm{pH}$; therefore, the system is not observable. However, because of the nature of the process, its dynamics is stable and the states are detectable. Therefore, it is possible to use a state estimator without the assumption of observability. ${ }^{33}$

At the beginning of the run, the true value of the state vector and the respective estimates are set according to

$$
\boldsymbol{x}(0)=\hat{\boldsymbol{x}}_{\mathrm{EKF}}(0)=\hat{\boldsymbol{x}}_{\mathrm{UKF}}(0)=\left[\begin{array}{l}
8.8 \times 10^{-4} \\
5.4 \times 10^{-4} \\
6.8 \times 10^{-4}
\end{array}\right] \mathrm{mol} / \mathrm{L}
$$

and the respective covariance matrixes,

$$
\hat{\boldsymbol{P}}_{\mathrm{EKF}}^{\mathrm{T}}(0)=\hat{\boldsymbol{P}}_{\mathrm{UKF}}^{\mathrm{T}}(0)=\operatorname{diag}\left\{\begin{array}{l}
0 \\
0 \\
0
\end{array}\right\} \mathrm{mol}^{2} / \mathrm{L}^{2}
$$

Although such a choice is obviously impossible in real systems, the motivation behind it in the present work is 2 -fold. On one hand, we expose both filters to the same consistent initial conditions and exclude from consideration the ad hoc solution of performance improvement by artificially increasing the initial covariance estimate. On the other hand, by choosing exact initial estimates, we avoid the transient behavior at the beginning of the simulation run, so that the associated errors do not mask the results of the remaining run. In fact, provided that an estimator is stable, in cases of continuous processes, the estimation errors during operation are of bigger importance than those that are caused by a wrong initial estimate. Nevertheless, the issue of convergence speed is addressed in the parameter estimation run described in Section 4.3.

Because of the system structure, the dimension of the augmented state vector is 7 and the additional parameter of the UKF, $\kappa$, is set to -4 .

The system is excited by a periodic changes in inlet flow $\left(q_{\mathrm{A}}\right)$, according to

$$
q_{\mathrm{A}}=1+0.06 \sin (0.04 t)
$$

where $t$ is time (given in minutes). The flow rate $q_{\mathrm{B}}$ is maintained at $0.265 \mathrm{~L} / \mathrm{min}$. Such operating conditions result in output $\mathrm{pH}$ between 6 and 4, which is the region of severe nonlinearity (Figure 6).

Two cases are considered: in Experiment I, the filters have access to the perfect model of the process, whereas in Experiment II, the model underestimates the system parameter $\theta$ by $1 \%$. To enable a fair comparison of the two filters, each experiment was repeated 450 times and
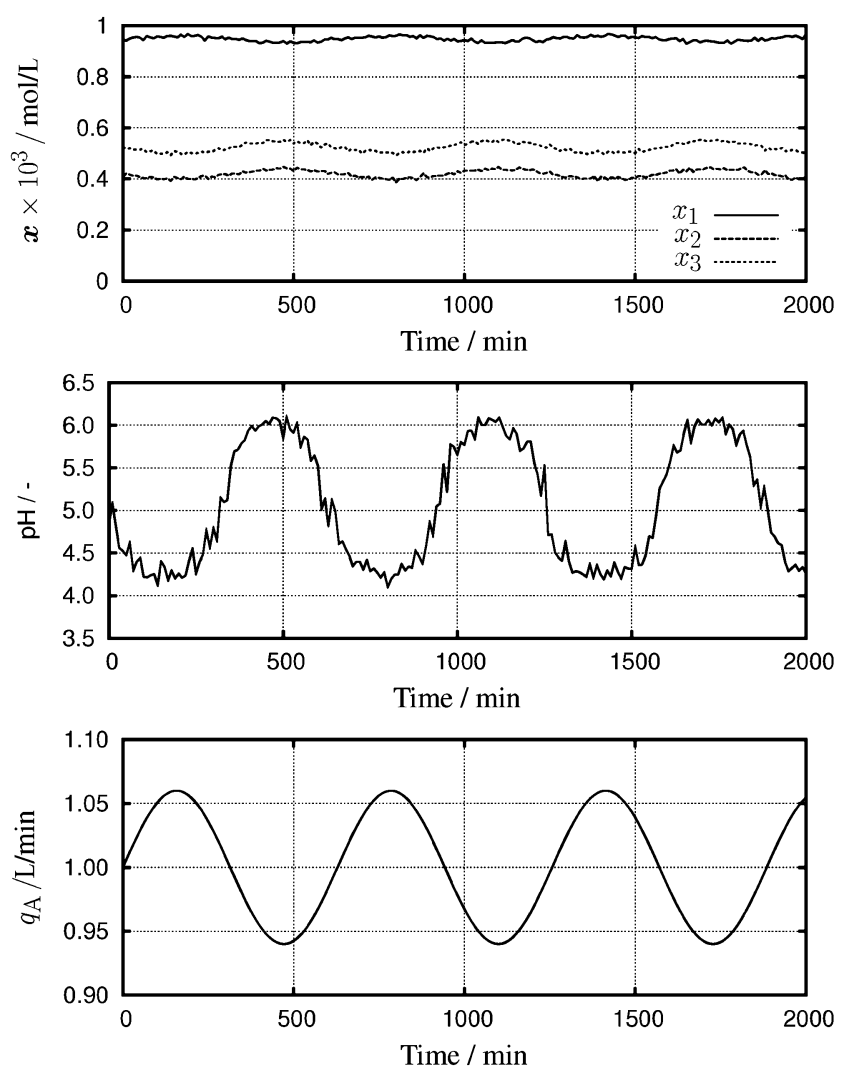

Figure 6. Open loop simulation results.

Table 3: Open-Loop Mean Square Estimation Errors over 450 Runs

\begin{tabular}{ccccc}
\hline method $^{a}$ & $x_{1}\left(\times 10^{-9}\right)$ & $x_{2}\left(\times 10^{-9}\right)$ & $x_{3}\left(\times 10^{-9}\right)$ & $y$ \\
\hline \multicolumn{5}{c}{ Experiment I: Perfect Model } \\
EKF & 2.7106 & 2.7089 & 2.7901 & $1.2448 \times 10^{-1}$ \\
$\mathrm{UKF}$ & 2.6999 & 2.6974 & 2.7789 & $8.4324 \times 10^{-2}$ \\
\multicolumn{5}{c}{ Experiment II: Model-Plant Mismatch } \\
EKF & 8.8688 & 5.3505 & 6.9480 & $1.0466 \times 10$ \\
$\mathrm{UKF}$ & 8.4134 & 5.0173 & 6.6868 & $0.9279 \times 10$ \\
${ }^{a} \mathrm{EKF}$ & $=$ & extended Kalman filter; UKF $=$ unscented Kalman \\
filter.
\end{tabular}

the estimation errors were averaged and presented in Figures 7 and 8, and in Table 3.

Although, in Experiment I, the process model is perfect, the states are corrupted by the system noise and the filters must address this by decreasing the incurred uncertainty by effective incorporation of the measurements into the state estimate. The relative weight in the final estimate of the a priori estimate, obtained from the model prediction, and the measurement is determined by the Kalman gain that, in turn, is dependent on the predicted output covariance and the innovation covariance. Because the output equation is nonlinear, even in this perfect case, the unscented Kalman filter produces better results (see Table 3), although the difference in state estimate error is not significant. However, because of the high nonlinearity of eq 25 , these insignificantly higher state estimation errors of the EKF, as compared with the ones of the UKF, incur tangible differences in the output variable prediction errors (see bottom plot of Figure 7).

Experiment II poses a more challenging task, as the model predictions have a bias, because of the error in the parameter $\theta$. A possible way of taking into account unmodeled features or model-plant mismatch is to increase the system noise covariance artificially. In this 

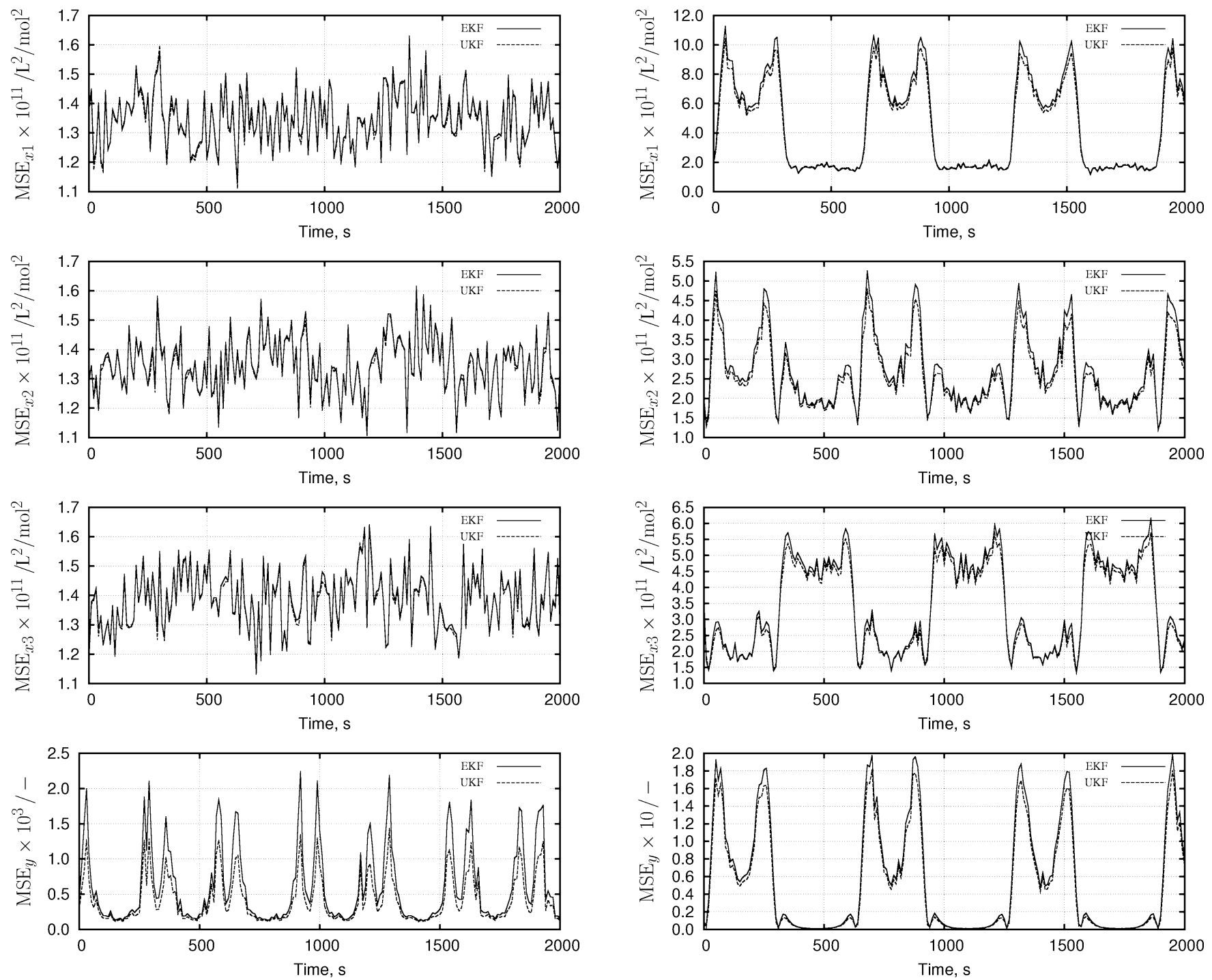

Figure 7. Results of experiment I: perfect parameters.

respect, this matrix is treated as a measure of confidence in the process model and not as the reflection of a system noise characteristic. Nevertheless, the focus in this work is to expose the filter to the same consistent information about the stochastic disturbances, and, therefore, the system noise covariance matrix is maintained, as in the previous case. Because model predictions contain an additional error caused by the mismodeling, the measurement is of greater importance to reduce the overall estimation error. Now that the UKF is able to predict better the respective covariances, the resulting state estimates are visibly better than those of the EKF (Figure 8).

4.3. Parameter Estimation. In the simulation given previously, the actual states of the system were estimated. However, a Kalman-type framework may be used to estimate model parameters. To test the two filters in this role, the estimation of parameter $K_{\mathrm{x}}$ is considered. Its dynamics is assumed to be of random walk nature. Moreover, it is subject to zero-mean white noise disturbance with a variance of $10^{-17} \mathrm{~mol}^{2} / \mathrm{L}^{2}$. Such variation may be caused by a random variation in the inlet temperatures that affects the tank temperature. The measurement is corrupted with zero-mean white noise with a variance of $10^{-3}$.

The aim of this simulation is to compare the speed of convergence of the two filters in a more-realistic situ-

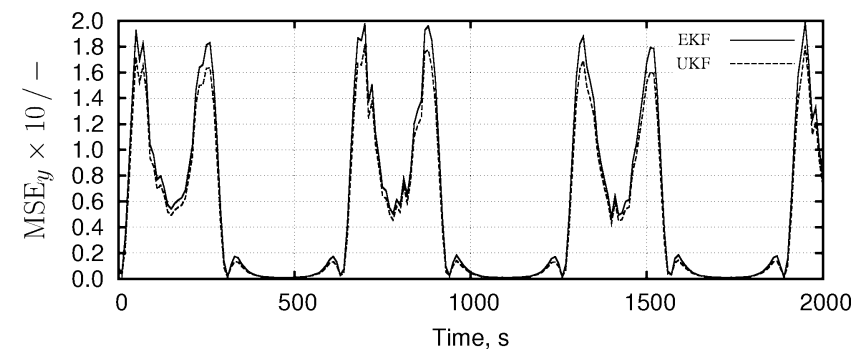

Figure 8. Results of experiment II: plant-model mismatch.

ation when the correct initial estimate is not available. Furthermore, the initial concentrations are chosen to be those resulting in $\mathrm{pH}=7$, whereas the flow rate $q_{\mathrm{B}}$ is set to $0.265 \mathrm{~L} / \mathrm{min}$, causing a nonlinear transition with a new steady-state $\mathrm{pH}$ of $\sim 5$. Although both filters have access to the perfect system model, the parameter dynamics is not stable, and, therefore, the present case is more challenging than that described previously.

The initial estimate of the parameter is $\hat{K}_{\mathrm{x}}(0)=7 \times$ $10^{-7} \mathrm{~mol} / \mathrm{L}$, and its true value is $K_{\mathrm{x}}(0)=10^{-6} \mathrm{~mol} / \mathrm{L}$.

The simulation is performed 5000 times, to provide a fair comparison. The mean-squared estimation errors (the difference between the true parameter value and its estimate) are represented in Figure 9. The speed of convergence of the UKF is higher than that of the EKF, and the steady-state error is lower. As in the state estimation case, the UKF did not need to evaluate the output function Jacobian.

\section{Conclusions}

In this work, we compared the performance of the extended Kalman filter (EKF) and unscented Kalman filter $(\mathrm{UKF})$ in a simulated $\mathrm{pH}$ process. The moreadvanced approximation of the mean and covariance of a random variable undergoing a nonlinear transformation enabled the UKF to yield more-accurate estimation 


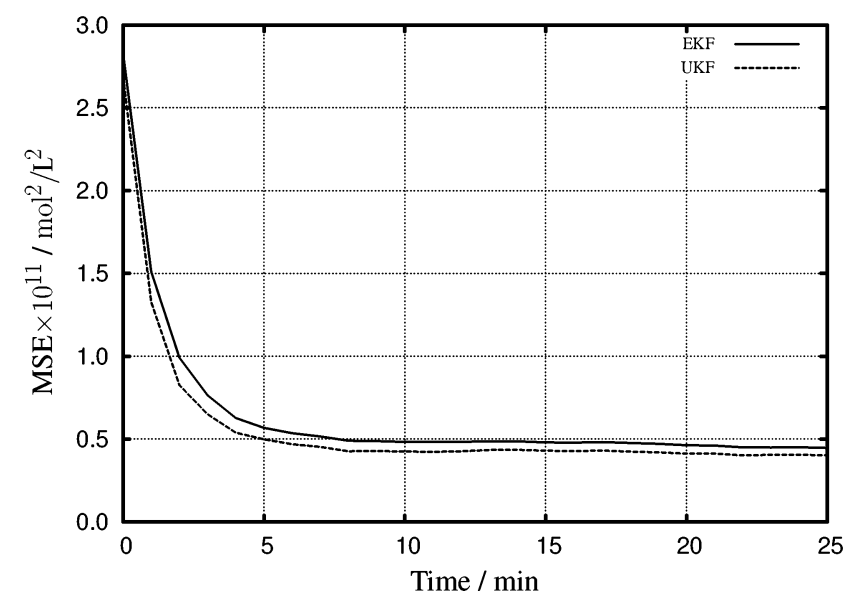

Figure 9. Mean-squared parameter estimation errors.

results than the EKF. It is also noteworthy that the Jacobian of the output equation is a complex expression that requires considerable computational burden. However, the UKF formulation needed neither its derivation nor its online evaluation.

Because of its easy implementation and superior performance, the UKF may be used as a drop-in replacement for the EKF, benefiting typical chemical process applications.

\section{Acknowledgment}

The first author is thankful to Fundação para a Ciência e a Tecnologia for his grant (PRAXIS XXI/BD/ 19609/99). The high-level computer language Octave and the plotting program Gnuplot, both of which are free software, were used in this work.

\section{Literature Cited}

(1) Soroush, M. State and Parameter Estimation and Their Application in Process Control. Comput. Chem. Eng. 1998, 23 (2), 229.

(2) Kalman, R. E. A New Approach to Linear Filtering and Prediction Problems. Trans. ASME, Ser. D 1960, 82, 34.

(3) Jazwinski, A. H. Stochastic Processes and Filtering Theory; Academic Press: New York, 1970.

(4) Moler, C.; Van Loan, C. Nineteen Dubious Ways to Compute the Exponential of a Matrix. SIAM Rev. 1978, 20, 801.

(5) Kayihan, A.; Doyle, F. J., III. Friction Compensation for a Process Control Valve. Control Eng. Pract. 2000, 8 (7), 799.

(6) Downs, J. J.; Vogel, E. F. A Plant-Wide Industrial Process Control Problem. Comput. Chem. Eng. 1993, 17 (3), 245.

(7) Schei, T. S. A Finite Difference Method for Linearization in Nonlinear Estimation Algorithms. Automatica 1997, 33 (11), 2053.

(8) Julier, S. J.; Uhlmann, J. K.; Durrant-Whyte, H. F. A New Approach for Filtering Nonlinear Systems. In Proceedings of the 1995 American Control Conference, Seattle, WA, 1995; American Automatic Control Council: Green Valley, AZ, 1995; p 1628.

(9) Julier, S. J.; Uhlmann, J.; Durrant-Whyte, H. F. A New Method for the Nonlinear Transformation of Means and Covariances in Filters and Estimators. IEEE Trans. Autom. Control 2000, 45 (3), 477.

(10) Nørgaard, M.; Poulsen, N. K.; Ravn, O. Advances in Derivative-Free State Estimation for Nonlinear Systems. Automatica 2000, 36 (11), 1627.

(11) Lefebvre, T.; Bruyninckx, H.; DeSchutter, J. Comment on "A New Method for the Nonlinear Transformation of Means and Covariances in Filters and Estimators". IEEE Trans. Autom. Control 2002, 47 (8), 1406.

(12) Julier, S. J.; Uhlmann, J. K. Unscented Filtering and Nonlinear Estimation. Proc. IEEE 2004, 92 (3), 401.
(13) Crassidis, J. L.; Markley, F. L. Unscented Filtering for Spacecraft Attitude Estimation. J. Guid. Control Dynam. 2003, 26 (4), 536.

(14) Ristic, B.; Farina, A.; Benvenuti, D.; Arulampalam, M. S. Performance Bounds and Comparison of Nonlinear Filters for Tracking a Ballistic Object on Re-entry. IEE Proc.-Radar Sonar Navig. 2003, 150 (2), 65.

(15) Popescu, C. A.; Wong, Y. S. Nonlinear Statistical Approach for Aeroelastic Response Prediction. J. Guid. Control Dynam. 2003, 26 (4), 565.

(16) Caffery, J.; Stuber, G. L. Nonlinear Multiuser Parameter Estimation and Tracking in CDMA Systems. IEEE Trans. Commun. 2000, 48 (12), 2053.

(17) Ristic, B.; Arulampalam, M. S. Tracking a Manuevering Target Using Angle-Only Measurements: Algorithms and Performance. Signal Process. 2003, 83 (6), 1223.

(18) Choi, J.; Lima, A. C. D.; Haykin, S. Unscented Kalman Filter-Trained Recurrent Neural Equalizer for Time-Varying Channels. In Proceedings of IEEE International Conference on Communications, May 11-15, 2003, Anchorage, AK; IEEE: New York, 2003; p 3241.

(19) Wan, E. A.; vander Merwe, R. The Unscented Kalman Filter for Nonlinear Estimation. In Proceedings of Symposium 2000 on Adaptive Systems for Signal Processing, Communication, and Control (AS-SPCC), October 1-4, 2000, Lake Louise, Alberta, Canada; IEEE: New York, 2000; p 153.

(20) Simon, L.; Karim, M. N. Control of Starvation-Induced Apoptosis in Chinese Hamster Ovary Cell Cultures. Biotechnol. Bioeng. 2002, 78 (6), 645 .

(21) Sitz, A.; Kurths, J.; Voss, H. U. Identification of Nonlinear Spatiotemporal Systems via Partitioned Filtering. Phys. Rev. E 2003, 68 (1)

(22) Griffin, C. D.; Croson, D. V.; Feeley, J. J. Kalman Filtering Applied to a Reagent Feed System. Chem. Eng. Progr. 1988, 84 (10), 45.

(23) Romanenko, A.; Castro, J. A. A. M. The Unscented Filter as an Alternative to the EKF for Nonlinear State Estimation: A Simulation Case Study. Comput. Chem. Eng. 2004, 28(3), 347.

(24) Sitz, A.; Schwarz, U.; Kurths, J.; Voss, H. U. Estimation of Parameters and Unobserved Components for Nonlinear Systems from Noisy Time Series. Phys. Rev. E 2002, 66 (1).

(25) Julier, S. J.; Uhlmann, J. K. A New Extension of the Kalman Filter to Nonlinear Systems. In Proceedings of AeroSense: The 11th International Symposium on Aerospace/Defense Sensing, Simulation and Controls; SPIE: Orlando, FL, 1997.

(26) van Zandt, J. R. A More Robust Unscented Transform. In Proceedings of the 13th Conference on Signal and Data Processing of Small Targets, San Diego, CA, July 30-August 2, 2001; SPIE: Bellingham, WA, 2001; p 371.

(27) Tenne, D.; Singh, T. The Higher Order Unscented Filter. In Proceedings of the 2003 American Control Conference, Denver, CO, 2003; IEEE: New York, 2003; p 2441.

(28) Julier, S. J.; Uhlmann, J. K. Reduced Sigma Point Filters for the Propagation of Means and Covariances through Nonlinear Transformations. In Proceedings of the 2002 American Control Conference, Anchorage, AK, 2002; IEEE: New York, 2002; p 887.

(29) Julier, S. J. The Scaled Unscented Transformation. In Proceedings of the 2002 American Control Conference, Anchorage, AK, 2002; IEEE: New York, 2002; p 4555.

(30) vander Merwe, R.; Wan, E. A. The Square-Root Unscented Kalman Filter for State and Parameter-Estimation. In Proceedings of 2001 IEEE International Conference on Acoustics, Speech, and Signal Processing, Salt Lake City, UT, May 7-11, 2001; IEEE: New York, 2001; p 3461.

(31) Biagiola, S. I.; Figueroa, J. L. State Estimation in Nonlinear Processes. Application to $\mathrm{pH}$ Process Control. Ind. Eng. Chem. Res. 2002, 41 (19), 4777.

(32) Grewal, M. S.; Andrews, A. P. Kalman Filtering: Theory and Practice; Prentice-Hall: Englewood Cliffs, NJ, 1993.

(33) Henson, M. A.; Seborg, D. E. Adaptive Nonlinear Control of a pH Neutralization Process. IEEE Trans. Control Syst. Technol. 1994, 2 (3), 169.

Received for review February 5, 2004 Revised manuscript received September 6, 2004 Accepted September 8, 2004 